\title{
Detecting a Target Location Using a Mobile Robot With Range Only Measurement
}

\author{
Gaurav Chaudhary \\ Systems and Control Engineering \\ Indian Institute of Technology, Bombay \\ Mumbai, India \\ Email: gauravch@sc.iitb.ac.in
}

\author{
Arpita Sinha \\ Systems and Control Engineering \\ Indian Institute of Technology, Bombay \\ Mumbai, India \\ Email: asinha@sc.iitb.ac.in
}

\begin{abstract}
This paper addresses the problem of guiding a mobile robot to a point of interest or the target point using only range sensors. The bearing information is not available. The target point can be the source of some gas leakage or nuclear radiation or it can be some landmark or beacon. A control strategy is proposed that can bring the robot arbitrarily closed to the target point. It is shown analytically that this strategy works for any initial condition of the robot with respect to the target point. Simulations are carried out to validate the results obtained in this paper.
\end{abstract}

\section{INTRODUCTION}

Strategy for autonomous robots to capture a stationary or a moving target using range only measurement has attracted special attention in field of robotics (see e.g. [1], [2], [3] and references therein). The objective of the paper is to find a strategy to capture a target with range only measurement. We can use this strategy in applications such as finding the point of leakage of some gas by only knowing the intensity of gas at the robot position or in pursuit evasion problem using only distance information between the pursuer and the evader.

Several target tracking approaches are proposed in the literature that uses only the range information to capture a stationary or moving target. In [1], a sliding mode control strategy is proposed using which the robot follows the target with constant speed while maintaining the predefine range margin from the target. In [2], guidance algorithms for following both steady and moving targets are proposed and the guidance methods have the property that the robot follows a trajectory that is close to a certain curve called equiangular spiral. A switched logicbased control strategy to solve the pursuit problem for target tracking is shown in [3]. This problem is solved in discrete time in [4] where the target position is estimated at each instance of time and the robot moves towards the estimated target position in discrete steps. In [5], local observability requirements are developed for target tracking and are verified by evaluating the performance of a state estimator. Problem of target motion analysis from range and range-rate measurements is investigated in [6]. Range only Extended Kalman Filter (EKF) is utilized to track the trajectory of the moving target in [7] and several different estimation based techniques are discussed in ( [8]-[12]) for tracking of target using range only measurement. For a team of mobile robots tracking a moving target using distance-only measurements, Zhou et al.
( [13], [14]) have proposed algorithms for determining the set of feasible locations that each robot should move to in order to collect the most informative measurements, that is, the distance measurements that minimize the uncertainty about the position of the target. A strategy for searching the source of gas using mobile robot is discussed in [15]. When the presence of gas is detected, the robot turns in the direction of the airflow that carries the gas and looks for any suspicious object. In [16], the robot is driven by the concentration gradient generated by a gas leak.

In this paper, we propose a generalized guidance strategy for detecting a stationary target using only range and rangerate information. The problem in continuous time is addressed. A single robot is used for the detection. The strategy does not involve any estimation of states. The robot can reach arbitrarily close to the target from any initial position.

This paper is organized as follows: The problem is define in Section II. In Section III, the mathematical analysis for locating the target is discussed. Simulation results for different cases are presented in Section IV. Section V concludes the paper.

\section{PROBLEM STATEMENT}

Consider a mobile robot that can measure the distance and the rate of change of distance from a given point. The problem is to guide the robot to that point. We consider an unicycle model for the robot, the kinematics of which is given by

$$
\begin{array}{r}
\dot{x_{r}}=v_{1} \cos (\alpha) \\
\dot{y_{r}}=v_{1} \sin (\alpha) \\
\dot{\alpha}=u
\end{array}
$$

where $\left(x_{r}, y_{r}\right)$ is the instantaneous position of the robot, $v_{1}$ is the linear velocity of the robot and $u$ is the control input to the robot. Thus, the angular velocity of the robot is controlled while the linear velocity is constant.

We are considering a stationary target point that the robot has to locate. Let the target point be at $\left(x_{t}, y_{t}\right)$ with respect to some reference frame and while the robot is at $\left(x_{r}(t), y_{r}(t)\right)$ at time $t$. This is shown in Figure 1. Let the distance between the robot and the target, that is, the line-of-sight (LOS) distance be $R$ and the LOS angle be $\theta$. The robot can measure $R$ and $\dot{R}$, but not $\theta$. Thus, $u$ is some function of $R$ and $\dot{R}$. We determine the conditions that this function needs to satisfy so that it can 


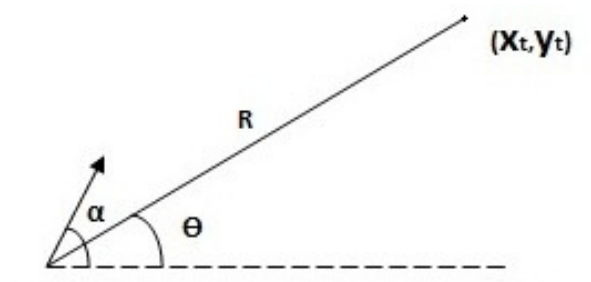

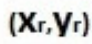

Reference

Fig. 1. Engagement geometry between the robot and the target point

steer the robot to the target. The conditions are derived in the next section. When the distance between the robot and the target is less than $R_{c}$, we assume that the target is captured. We call $R_{c}$ the capture radius and $R_{c}>0$.

\section{ANALYSIS}

Consider Figure 1. The line-of-sight (LOS) between the robot at $\left(x_{r}, y_{r}\right)$ and the target at $\left(x_{t}, y_{t}\right)$ is characterized by

$$
\begin{aligned}
& V_{R}=\dot{R}=-v_{1} \cos (\alpha-\theta) \\
& V_{\theta}=R \dot{\theta}=-v_{1} \sin (\alpha-\theta)
\end{aligned}
$$

where $V_{R}$ is the relative velocity component with respect to the robot along the $\operatorname{LOS}$ and $V_{\theta}$ is the relative velocity component with respect to the robot perpendicular to the LOS. Differentiating the equation (4) and (5) with respect to time,

$$
\begin{gathered}
\dot{V}_{R}=v_{1} \sin (\alpha-\theta)(\dot{\alpha}-\dot{\theta}) \\
\dot{V}_{\theta}=-v_{1} \cos (\alpha-\theta)(\dot{\alpha}-\dot{\theta})
\end{gathered}
$$

which can be rewritten, using (4) and (5), as

$$
\begin{gathered}
\dot{V}_{R}=-V_{\theta}\left(\dot{\alpha}-\frac{V_{\theta}}{R}\right) \\
\dot{V}_{\theta}=V_{R}\left(\dot{\alpha}-\frac{V_{\theta}}{R}\right)
\end{gathered}
$$

Squaring and adding equation (6) and (7), we get

$$
V_{R}^{2}+V_{\theta}^{2}=v_{1}^{2}
$$

In $\left(V_{\theta}, V_{R}\right)$ plane, Eqn. (10) implies a circle with center at the origin and radius $v_{1}$, which is shown in Figure 2. Thus, the motion of the robot has to be such that the instantaneous $\left(V_{\theta}, V_{R}\right)$ point will always lie on this circle. We assume that the target is captured when $R \leq R_{c}$. The following theorem states the conditions under which capture is possible.

Theorem 1: A robot, with kinematics (1)-(3), will be able to capture a stationary target from any initial position if it uses a control law given by

$$
u=f\left(V_{R}\right)
$$

where $f\left(V_{R}\right)$ is some function of $V_{R}$ which satisfies

$$
\begin{gathered}
f\left(V_{R}\right)\left\{\begin{array}{cl}
=0, & V_{R}=-v_{1} ; \\
>0, & V_{R} \in\left(-v_{1}, 0\right) ; \\
>\frac{v_{1}}{R_{c}}, & V_{R} \in\left[0, v_{1}\right] .
\end{array}\right. \\
\frac{d f\left(V_{R}\right)}{d V_{R}}>0, \quad V_{R} \in\left(-v_{1}, v_{1}\right) ;
\end{gathered}
$$

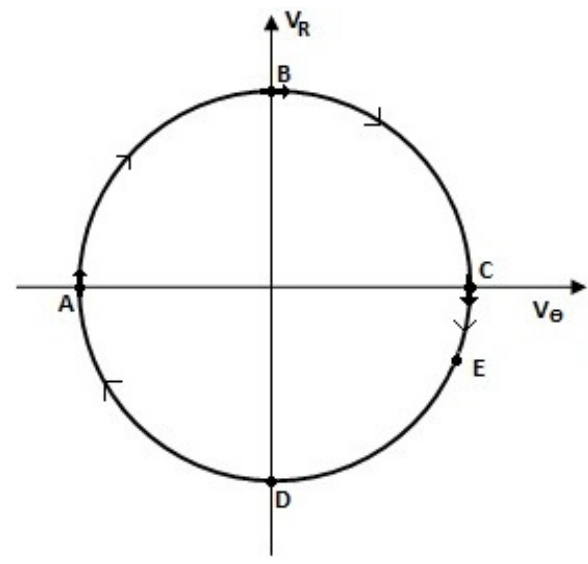

Fig. 2. Engagement trajectory in $\left(V_{\theta}, V_{R}\right)$ space

Proof: In order to capture the target, $R$ should decrease, which implies $V_{R}<0$. From Figure $2,\left(V_{\theta}, V_{R}\right)$ always moves on the circle. We study the movement of $\left(V_{\theta}, V_{R}\right)$ when the control law (11) is applied to the robot.

From (13), $u$ is an increasing function with respect to $V_{R}$ for $V_{R} \in\left(-v_{1}, v_{1}\right)$. From (8),(9) and (11),

$$
\begin{gathered}
\dot{V}_{R}=-V_{\theta}\left(f\left(V_{R}\right)-\frac{V_{\theta}}{R}\right) \\
\dot{V}_{\theta}=V_{R}\left(f\left(V_{R}\right)-\frac{V_{\theta}}{R}\right)
\end{gathered}
$$

Let us consider that at time $t=0,\left(V_{\theta}, V_{R}\right)$ lies on different sectors of the circle in Figure 2.

Case 1: Between points $D$ and $A$

In this region, $-v_{1}<V_{R}<0$ and $-v_{1}<V_{\theta}<0$. Let $V_{R}=-\beta v_{1}$ where $\beta \in(0,1)$. Then, from (10), $V_{\theta}=$ $-v_{1} \sqrt{1-\beta^{2}}<0$ and $f\left(V_{R}\right)>0$. From (14) and (15)

$$
\begin{array}{r}
\dot{V}_{R}=v_{1} \sqrt{1-\beta^{2}}\left(f\left(V_{R}\right)+\frac{v_{1} \sqrt{1-\beta^{2}}}{R}\right) \\
\dot{V}_{\theta}=-v_{1} \beta\left(f\left(V_{R}\right)+\frac{v_{1} \sqrt{1-\beta^{2}}}{R}\right)
\end{array}
$$

which implies $\dot{V}_{R}>0$ and $\dot{V}_{\theta}<0$. Thus, the point $\left(V_{\theta}, V_{R}\right)$ in this region will move as shown in Figure 2.

Case 2: At point $A$

We have $V_{R}=0, V_{\theta}=-v_{1}$ and $f\left(V_{R}\right)>\frac{v_{1}}{R_{c}}>0$. From (14) and (15),

$$
\begin{array}{r}
\dot{V}_{R}=v_{1}\left(f\left(V_{R}\right)+\frac{v_{1}}{R}\right) \\
\dot{V}_{\theta}=0
\end{array}
$$

which implies $\dot{V}_{R}>0$ and the movement of $\left(V_{\theta}, V_{R}\right)$ point is as shown in Figure 2.

Case 3: Between points $A$ and $B$

In this region $0<V_{R}<v_{1}$ and $-v_{1}<V_{\theta}<0$. Assuming $V_{R}=v_{1} \beta>0$ for some $\beta \in(0,1), V_{\theta}=-v_{1} \sqrt{1-\beta^{2}}<0$ 
and $f\left(V_{R}\right)>0$. From (14) and (15)

$$
\begin{array}{r}
\dot{V}_{R}=+v_{1} \sqrt{1-\beta^{2}}\left(f\left(V_{R}\right)+\frac{v_{1} \sqrt{1-\beta^{2}}}{R}\right) \\
\dot{V}_{\theta}=v_{1} \beta\left(f\left(V_{R}\right)+\frac{v_{1} \sqrt{1-\beta^{2}}}{R}\right)
\end{array}
$$

which implies $\dot{V}_{R}>0$ and $\dot{V}_{\theta}>0$ and the movement of $\left(V_{R}, V_{\theta}\right)$ point is as shown in Figure 2.

Case 4: At point $B$

Here, $V_{R}=v_{1}, V_{\theta}=0$ and $f\left(V_{R}\right)>0$. From (14) and (15)

$$
\begin{array}{r}
\dot{V}_{R}=0 \\
\dot{V}_{\theta}=v_{1} f\left(V_{R}\right)
\end{array}
$$

Hence, $\dot{V}_{\theta}>0$ and the movement of $\left(V_{R}, V_{\theta}\right)$ point is as shown in Figure 2.

Case 5: Between point $B$ and $C$

In this region, $0<V_{R}<v_{1}$ and $0<V_{\theta}<v_{1}$. Again, let $V_{R}=v_{1} \beta>0$ for some $\beta \in(0,1)$. Then $V_{\theta}=v_{1} \sqrt{1-\beta^{2}}>$ 0 and $f\left(V_{R}\right)>\frac{v_{1}}{R_{c}}$. From (14) and (15)

$$
\begin{array}{r}
\dot{V}_{R}=-v_{1} \sqrt{1-\beta^{2}}\left(f\left(V_{R}\right)-\frac{v_{1} \sqrt{1-\beta^{2}}}{R}\right) \\
\dot{V}_{\theta}=v_{1} \beta\left(f\left(V_{R}\right)-\frac{v_{1} \sqrt{1-\beta^{2}}}{R}\right)
\end{array}
$$

Since, in this region, $f\left(V_{R}\right)>\frac{v_{1}}{R_{c}}$

$$
\left(f\left(V_{R}\right)-\frac{v_{1} \sqrt{1-\beta^{2}}}{R}\right)>\left(\frac{v_{1}}{R_{c}}-\frac{v_{1} \sqrt{1-\beta^{2}}}{R}\right)>0
$$

Hence, $\dot{V}_{R}<0$ and $\dot{V}_{\theta}>0$ and the movement of $\left(V_{R}, V_{\theta}\right)$ point is as shown in Figure 2.

Case 6: At point $C$

$V_{R}=0, V_{\theta}=v_{1}$ and $f\left(V_{R}\right)>\frac{v_{1}}{R_{c}}>0$. From (14) and (15)

$$
\begin{array}{r}
\dot{V}_{R}=-v_{1}\left(f\left(V_{R}\right)-\frac{v_{1}}{R}\right) \\
\dot{V_{\theta}}=0
\end{array}
$$

Since $f\left(V_{R}\right)>\frac{v_{1}}{R_{c}}$, using a similar argument as in the previous case

$$
\left(f\left(V_{R}\right)-\frac{v_{1}}{R}\right)>0
$$

Hence, $\dot{V}_{R}<0$ and the movement of $\left(V_{R}, V_{\theta}\right)$ point is as shown in Figure 2.

Case 7: Between points $C$ and $D$

In this region, $-v_{1}<V_{R}<0$ and $0<V_{\theta}<v_{1}$. Assuming $V_{R}=-v_{1} \beta>0$ for some $\beta \in(0,1), V_{\theta}=v_{1} \sqrt{1-\beta^{2}}>0$, and $f\left(V_{R}\right)>0$. From (14) and (15)

$$
\begin{array}{r}
\dot{V}_{R}=-v_{1} \sqrt{1-\beta^{2}}\left(f\left(-v_{1} \beta\right)-\frac{v_{1} \sqrt{1-\beta^{2}}}{R}\right) \\
\dot{V}_{\theta}=-v_{1} \beta\left(f\left(-v_{1} \beta\right)-\frac{v_{1} \sqrt{1-\beta^{2}}}{R}\right)
\end{array}
$$

Let

$$
g(\beta)=\left(f\left(-v_{1} \beta\right)-\frac{v_{1} \sqrt{1-\beta^{2}}}{R_{c}}\right)
$$

If $g(\beta)>0$ then $\dot{V}_{R}<0$ and $\dot{V}_{\theta}<0$ for all $R \geq R_{c}$. Since $f\left(V_{R}\right)$ is an increasing function, there exists a $\beta=\beta^{\prime}$ (say) such that $g(\beta)>0, \forall \beta \in\left(0, \beta^{\prime}\right]$. Let $E$ corresponds to the point $V_{R}=-v_{1} \beta^{\prime}$. In the region between $C$ to $E, g(\beta)>0$ and thus $\dot{V}_{R}<0$ and $\dot{V}_{\theta}<0 \forall R \geq R_{c}$. Hence, the movement of $\left(V_{\theta}, V_{R}\right)$ between $C$ and $E$ is as shown in Figure 2.

For a $\left(V_{\theta}, V_{R}\right)$ point within $E$ to $D$, nothing can be said about direction of movement of the point. However, it cannot leave this region because at $E$, if $R>R_{c}, \dot{V}_{R}<0$ and this will force the point to remain within the region. At $D, V_{R}=0$ and $V_{\theta}=0$, which is a stationary point and is discussed in the next case.

Case 8: At point $D$

$V_{R}=-v_{1}, V_{\theta}=0$ and $f\left(V_{R}\right)>\frac{v_{1}}{R_{c}}>0$. From equation (14) and (15)

$$
\begin{aligned}
\dot{V}_{R} & =0 \\
\dot{V}_{\theta} & =0
\end{aligned}
$$

Thus, $\left(V_{R}, V_{\theta}\right)$ is a stationary point and will not move. Since, $V_{\theta}=0$, the LOS angle do not change with time and $V_{R}<0$, the LOS distance keeps on decreasing. This corresponds to the case when the robot is heading directly to the target location.

From the different cases discussed above, it can be seen that, irrespective of the initial position of the robot with respect to the target, the $\left(V_{\theta}, V_{R}\right)$ point will always end up in the region between $E$ to $D$. Exceptions will occur when $R$ becomes less than $R_{c}$ before the point $E$ is reached. This can happen in the regions $D$ to $A$ and $C$ to $E$, when $V_{R}<0$ and this implies that capture has occurred. When the $\left(V_{\theta}, V_{R}\right)$ point is within $E$ to $D, V_{R}<0$ and thus the distance between the robot and the target will keep on decreasing until capture occurs.

Remark 1: Since $V_{R}$ and $v_{1}$ is known, from equation 4 , we can calculate the values of $(\alpha-\theta)$, but we do not use it because

1) We have to estimate the actual value of $(\alpha-\theta)$ from the two values obtained from (4).

2) The resulting control law may require switching based on the value of $V_{R}$. A switching control law is difficult to implement and results in chattering in practical implementations.

Remark 2: The capture is defined when $R \leq R_{c}$. Thus, by properly selecting $R_{c}$, the robot can be made to come arbitrarily close to the target. However, the control law (11) has to be changed appropriately.

\section{EXAMPLES AND SIMULATION RESULTS}

We present some candidate functions that can be used as the control input to the robot.

\section{A. Quadratic Function}

$$
f\left(V_{R}\right)=V_{R}^{2}+k_{1} V_{R}+k_{2}
$$

with

$$
\begin{array}{r}
k_{2}=\max \{a, b\} \\
k_{1}=v_{1}+\frac{k_{2}}{v_{1}}
\end{array}
$$




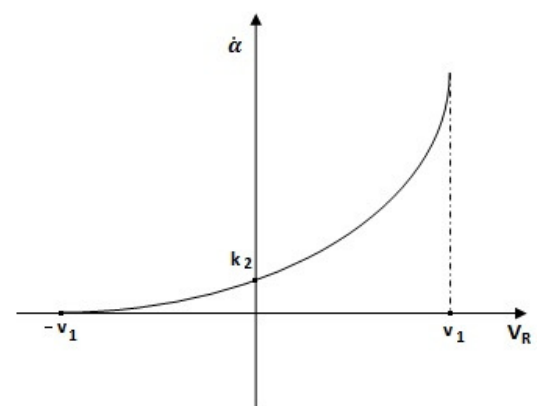

Fig. 3. A candidate quadratic control input function

where $a \geq v_{1}^{2}$ and $b>\frac{v_{1}}{R_{c}}$

It can be verified that this function (shown in Figure 3) satisfies (12) and (13). If we assume $v_{1}=10$ and $R_{c}=0.2$, then we can have

$$
\dot{\alpha}=f\left(V_{R}\right)=V_{R}^{2}+20 V_{R}+100
$$

For this function, $\beta^{\prime}=0.3106$ and corresponding value of $V_{R}=-3.106$.

\section{B. Linear function}

$$
f\left(V_{R}\right)=k_{1} V_{R}+k_{2}
$$

with

$$
\begin{aligned}
k_{2}>\frac{v_{1}}{R_{c}} \\
k_{1}=\frac{k_{2}}{v_{1}}
\end{aligned}
$$

This function also satisfies (12) and (13). Assuming again $v_{1}=$ 10 and $R_{c}=0.2$, we can have

$$
f\left(V_{R}\right)=6 V_{R}+60
$$

and then $\beta^{\prime}=0.18031$ and corresponding value of $V_{R}=$ -1.8031 .

We carried out simulations when the robot is following quadratic and linear control laws. The target is assumed to be at the origin and the different initial positions of the robot are considered. The initial position of the robot and the time taken to reach the target is tabulated in Table I. The initial conditions are selected such that each case corresponds to the different sectors in Figure 2. Also the initial distance between the target and the robot is same for all the cases. The variation of $R$ with respect to time is plotted in Figure 4 and 5 for the quadratic and linear control respectively.

We observe from Figure 4 and 5 that, in all the cases, $R$ decreases steadily for most of the time. However, there are instances when it also increases. This increase corresponds to the $\left(V_{R}, V_{\theta}\right)$ point begin in the region $A$ to $C$ in Figure 2. Since we have considered initial conditions in different sectors in Figure 2, hence the bump occurs at different time. In some cases, it is at the beginning, in some other, it is in between, while there are cases when it does not occur at all.
Consider a particular case when the robot is at a position given in Case 1 in Table I. For the control law given in (42), the trajectory of the robot is shown in the Figure 6. It is observed that the target has been captured. The variation of $V_{R}$ with respect to time is shown in Figure 7. It can be seen that $V_{R}<0$ for most of the time except for $t \in(0.1,0.5)$, where there is a rapid change in $V_{R}$. This corresponds to the sector between $A$ to $C$ in Figure 2. In this time, $R$ increases, which implies the distance between the robot and the target increases. However, with higher $V_{R}$, the control law is also high, which makes the robot change its heading very fast. As a result, the robot soon starts moving towards the target.

\section{CONCLUSIONS}

In the paper, we present a strategy that can bring a robot arbitrarily close to a target point when the robot can only measure the range and the range rate but has no information about the bearing angle to the target. The strategy is presented in a general form and is a function of rate of change of the distance to the target. We have derived the conditions that this function should satisfy. Two sample functions are presented. When the robot is using these functions as the control law, it is shown in simulation that the robot can capture the target, thus validating our analysis. This work can be extended to a moving target, which is under investigation. Also an estimation of the time taken to reach the target is an interesting avenue to be explore.

\section{REFERENCES}

[1] A. Matveev, H. Teimoori, and A. Savkin, "The problem of target following based on range-only measurements for car-like robots," in Decision and Control, 2009 held jointly with the 2009 28th Chinese Control Conference. CDC/CCC 2009. Proceedings of the 48th IEEE Conference on, dec. 2009, pp. $8537-8542$.

[2] H. Teimoori and A. Savkin, "Equiangular navigation and guidance of a wheeled mobile robot using range-only measurements," in Decision and Control, 2008. CDC 2008. 47th IEEE Conference on, dec. 2008, pp. $1747-1752$.

[3] Omid Namaki-Shoushtari, A. Pedro Aguiar, and Ali Khaki-Sedigh, "Target tracking of autonomous robotic vehicles using range-only measurements: a switched logic-based control strategy," International Journal of Robust and Nonlinear Control, 2011.

[4] S. Bopardikar, F. Bullo, and J. Hespanha, "A pursuit game with rangeonly measurements," in Decision and Control, 2008. CDC 2008. 47th IEEE Conference on, dec. 2008, pp. $4233-4238$.

[5] T. L. Song, "Observability of target tracking with range-only measurements," Oceanic Engineering, IEEE Journal of, vol. 24, no. 3, pp. 383 -387, jul 1999.

[6] Branko Ristic, Sanjeev Arulampalam, and James hlcCarthy, "Target motion analysis using range-only measurements: algorithms,performance and application," 2001.

[7] B. Fang, S. Wu, and G. Wei, "Vector miss distance measurement based on range-only target tracking," in Radar, 2006. CIE '06. International Conference on, oct. 2006, pp. 1 -4.

[8] A. Gadre and D. Stilwell, "Toward underwater navigation based on range measurements from a single location," in Robotics and Automation, 2004. Proceedings. ICRA '04. 2004 IEEE International Conference on, vol. 5, april-1 may 2004, pp. 4472 - 4477 Vol.5.

[9] E. Mazomenos, J. Reeve, and N. White, "Tracking with range-only measurements using a network of wireless sensors," in Broadband Communications, Networks, and Systems, 2009. BROADNETS 2009. Sixth International Conference on, sept. 2009, pp. $1-8$.

[10] G. Huang, K. Zhou, N. Trawny, and S. Roumeliotis, "A bank of maximum a posteriori estimators for single-sensor range-only target tracking," in American Control Conference (ACC), 2010, 30 2010-july 2 2010, pp. $6974-6980$. 


\begin{tabular}{|c|c|c|c|c|c|c|c|c|c|}
\hline Cases & $\begin{array}{c}1 \\
(\mathrm{D})-(\mathrm{A})\end{array}$ & $\begin{array}{c}2 \\
\text { at }(\mathrm{A})\end{array}$ & $\begin{array}{c}3 \\
(\mathrm{~A})-(\mathrm{B})\end{array}$ & $\begin{array}{c}4 \\
\text { at }(\mathrm{B})\end{array}$ & $\begin{array}{c}5 \\
(\mathrm{~B})-(\mathrm{C})\end{array}$ & $\begin{array}{c}6 \\
\text { at }(\mathrm{C})\end{array}$ & $\begin{array}{c}7 \\
(\mathrm{C})-(\mathrm{E})\end{array}$ & $\begin{array}{c}8 \\
(\mathrm{E})-(\mathrm{D})\end{array}$ & $\begin{array}{c}9 \\
\text { at }(\mathrm{D})\end{array}$ \\
\hline$x_{r_{0}}$ & 15 & 14.69 & -10 & -17.68 & -10 & 15.04 & 18 & 22 & 17.68 \\
$y_{r_{0}}$ & -20 & 20.22 & 22.91 & 17.68 & 22.91 & -19.96 & -17.34 & 11.87 & 17.68 \\
$\alpha_{0}$ & $135^{\circ}$ & $-36^{\circ}$ & $45^{\circ}$ & $135^{\circ}$ & $130^{\circ}$ & $37^{\circ}$ & $50^{\circ}$ & $150^{\circ}$ & $-135^{\circ}$ \\
$V_{R_{0}}$ & -9.89 & 0 & 3.65 & 10 & 9.59 & 0 & -0.68 & -5.24 & -10 \\
$V_{\theta_{0}}$ & -1.41 & -10 & -9.30 & 0 & 2.83 & 10 & 9.97 & 8.51 & 0 \\
\hline$t_{q c}$ & 2.74 & 2.73 & 2.73 & 2.72 & 2.72 & 2.71 & 2.71 & 2.71 & 2.48 \\
\hline$t_{l c}$ & 2.58 & 2.56 & 2.55 & 2.53 & 2.53 & 2.50 & 2.50 & 2.50 & 2.48 \\
\hline
\end{tabular}

TABLE I

INITIAL CONDITIONS AND TIME TO CAPTURE FOR QUADRATIC AND LINEAR CONTROL INPUT

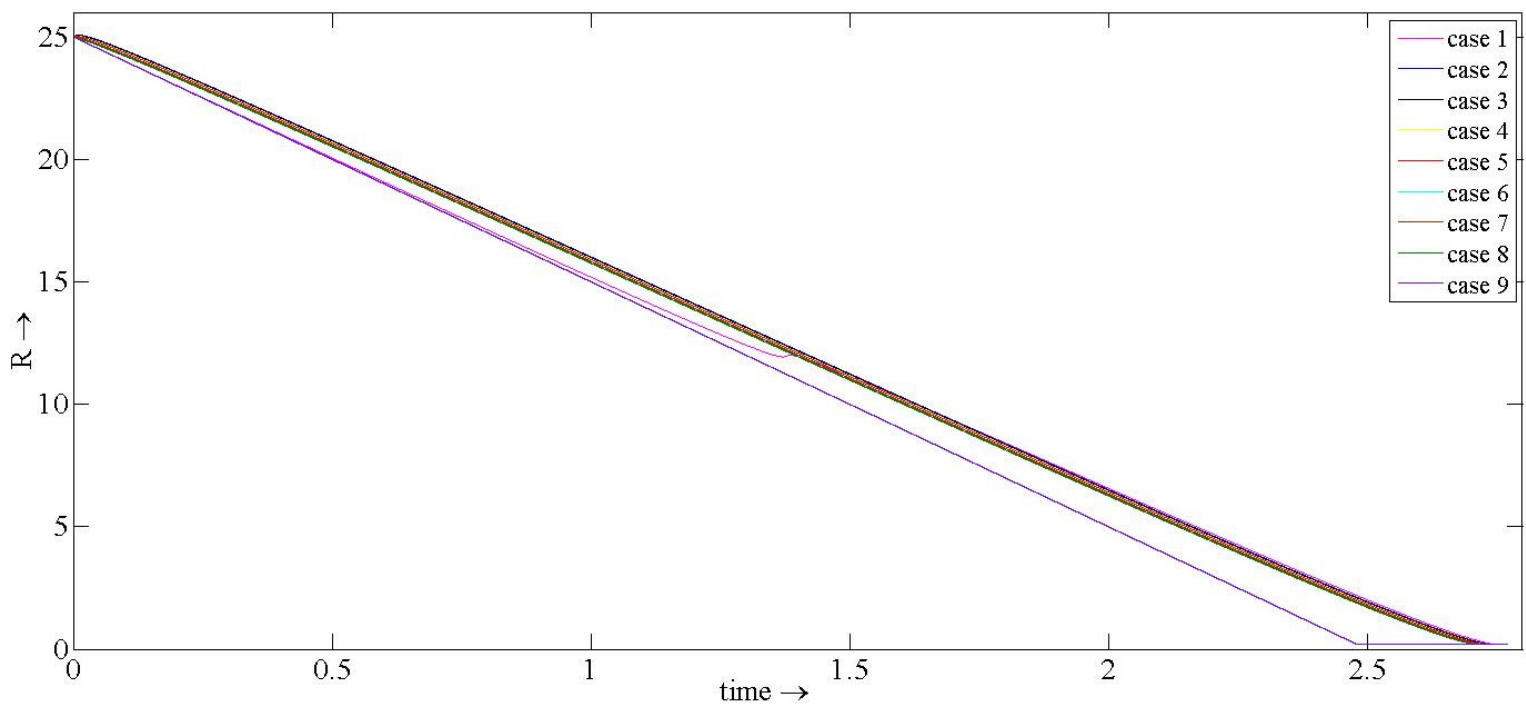

Fig. 4. Range v/s time for all the cases given in Table I for quadratic control input

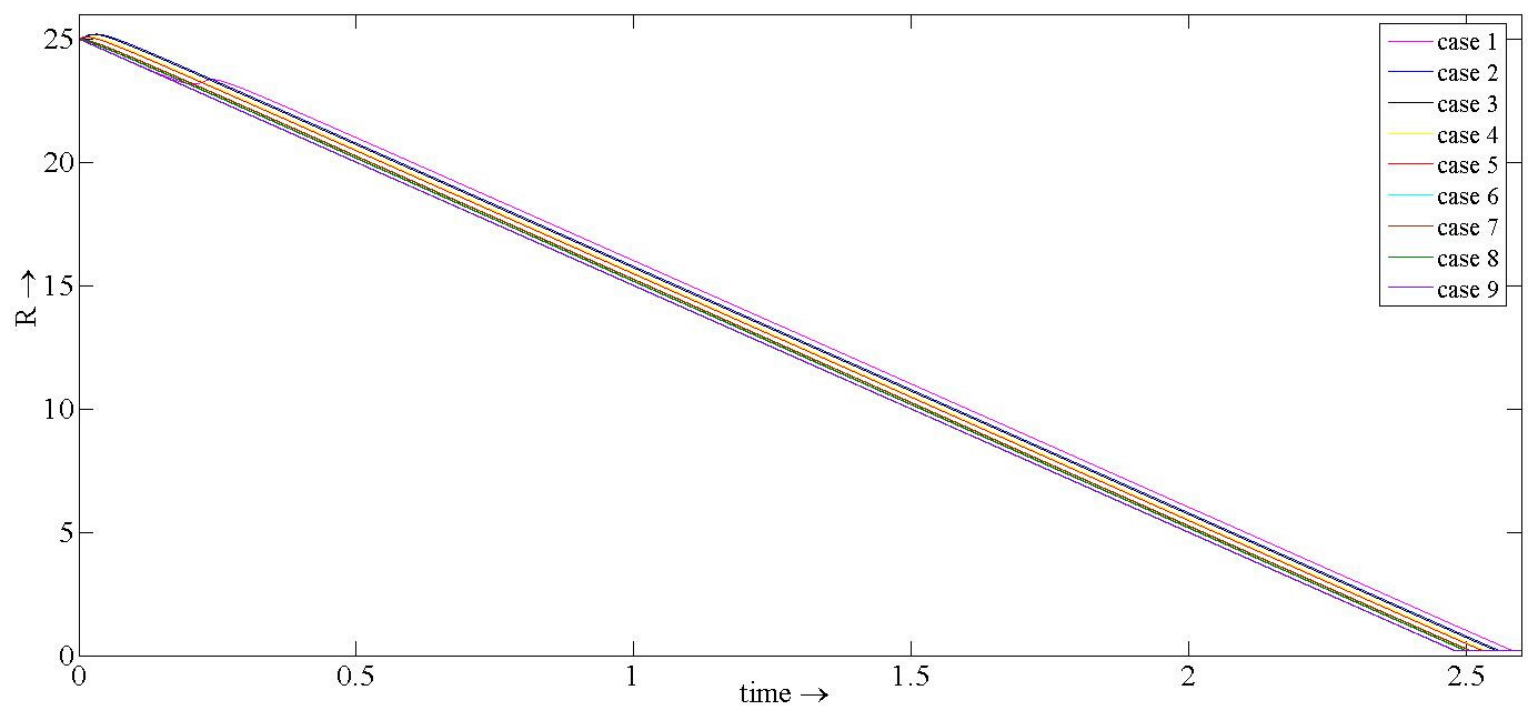

Fig. 5. $\mathrm{R} v / \mathrm{s}$ time for all the cases given in Table $\mathrm{I}$ for linear control input 


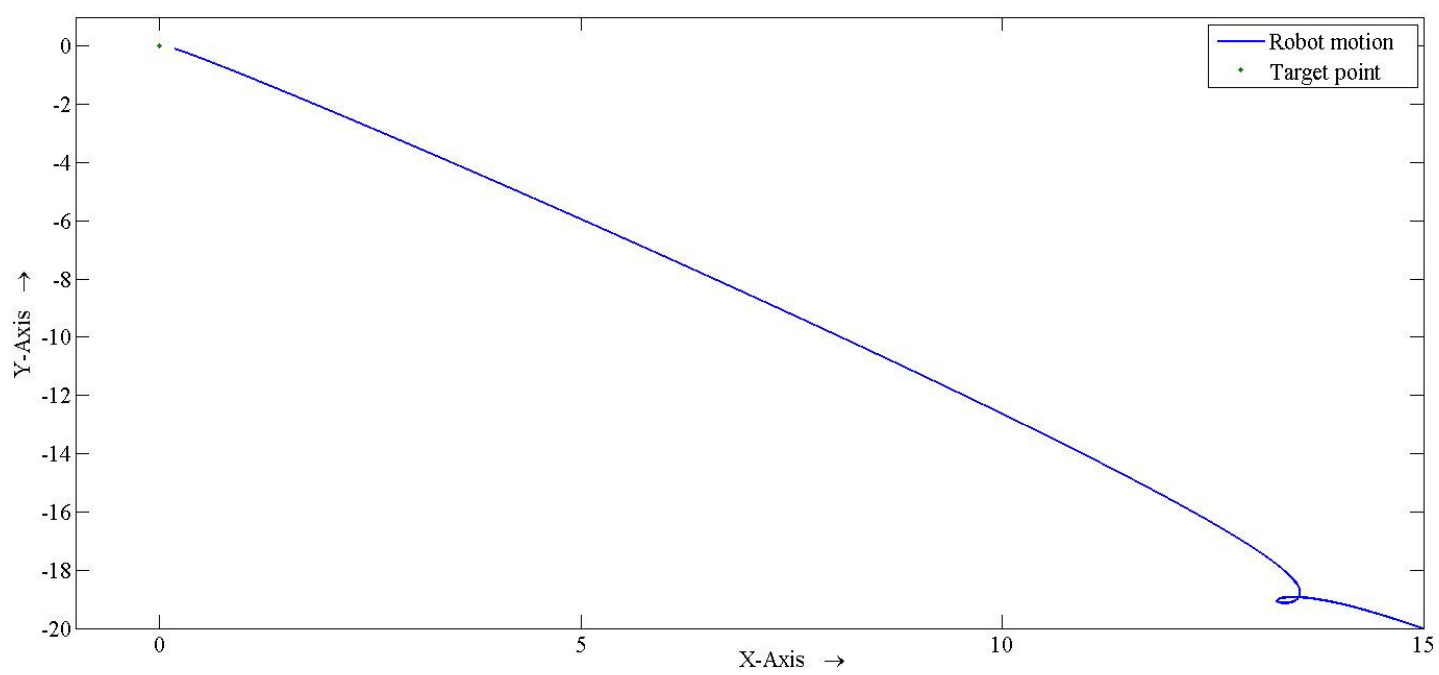

Fig. 6. Trajectory of robot for linear control input

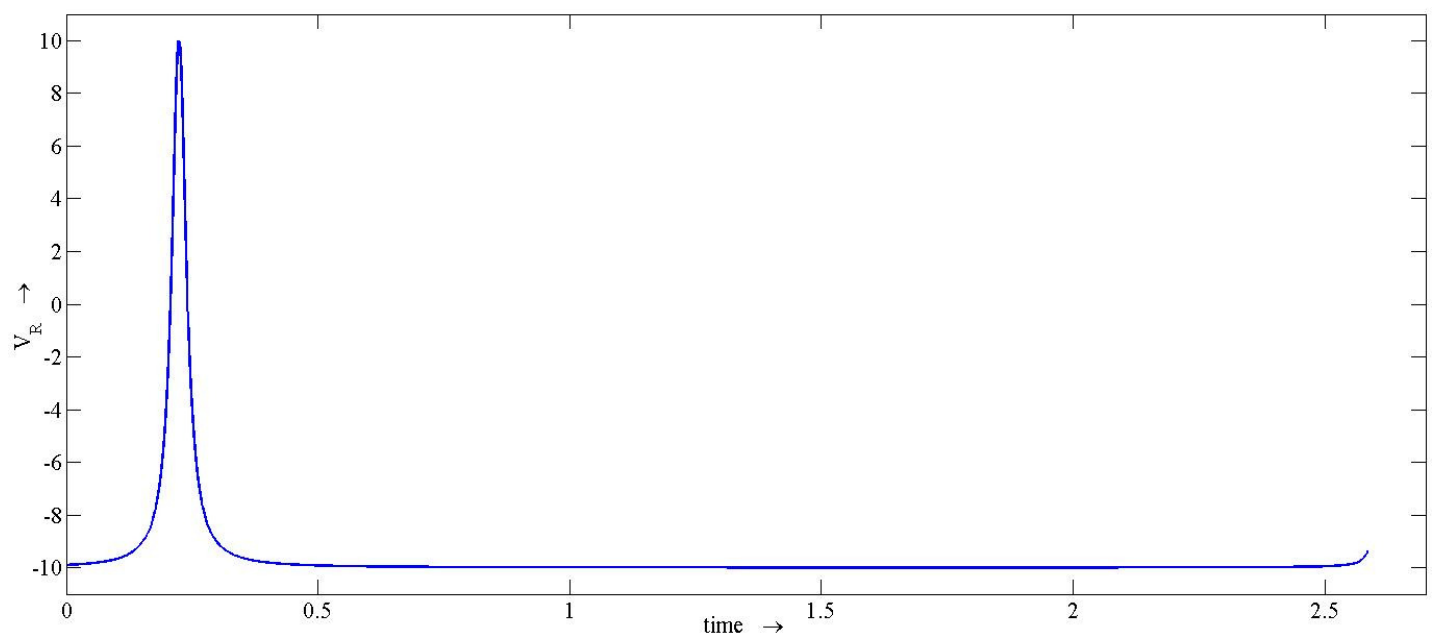

Fig. 7. $\quad V_{R} \mathrm{v} / \mathrm{s}$ time for linear control input

[11] B. Fang and S. Wu, "Angle-parameterizations range-only target tracking for scalar miss distance measurement system," in Signal Processing, 2006 8th International Conference on, vol. 1, 16-20 2006.

[12] H. Kameda, S. Tsujimichi, and Y. Kosuge, "Target tracking under dense environments using range rate measurements," in SICE '98. Proceedings of the 37th SICE Annual Conference. International Session Papers, jul 1998, pp. $927-932$.

[13] K. Zhou and S. Roumeliotis, "Optimal motion strategies for range-only distributed target tracking," in American Control Conference, 2006, june 2006, p. 6 pp.

[14] _ _ "Optimal motion strategies for range-only constrained multisensor target tracking," Robotics, IEEE Transactions on, vol. 24, no. 5, pp. 1168 -1185 , oct. 2008.

[15] H. Ishida, T. Ushiku, S. Toyama, H. Taniguchi, and T. Moriizumi, "Mobile robot path planning using vision and olfaction to search for a gas source," in Sensors, 2005 IEEE, 30 2005-nov. 3 2005, p. 4 pp.
[16] G. Sandini, G. Lucarini, and M. Varoli, "Gradient driven self-organizing systems," in Intelligent Robots and Systems '93, IROS '93. Proceedings of the 1993 IEEE/RSJ International Conference on, vol. 1, jul 1993, pp. $429-432$ vol.1. 